\title{
Towards 20 A negative hydrogen ion beams for up to 1 hour: Achievements of the ELISE test facility ${ }^{\text {a) }}$
}

\author{
U. Fantz ${ }^{\text {b) }}$, B. Heinemann, D. Wünderlich, R. Riedl, W. Kraus, R. Nocentini, and F. \\ Bonomo
}

${ }^{1}$ Max-Planck-Institut für Plasmaphysik, Boltzmannstr. 2, 85748 Garching, Germany

The large-scale RF-driven ion source of the test facility ELISE is aimed to deliver an accelerated ion current of $20 \mathrm{~A} \mathrm{D}^{-}\left(23 \mathrm{~A} \mathrm{H}^{-}\right)$with an extracted electron-to-ion ratio below one for up to one hour. Since the first plasma pulses for $20 \mathrm{~s}$ in volume operation in Early 2013, followed by caesiation of the ion source, substantial progress has been achieved in extending the pulse length and the RF power. The record pulses in hydrogen are: stable $400 \mathrm{~s}$ pulses with an extracted ion current of $18.3 \mathrm{~A}$ at $180 \mathrm{~kW}$ total RF power and $9.3 \mathrm{~A}$ at $80 \mathrm{~kW}$ stable for one hour. For deuterium pulse length and RF power are limited by the amount of coextracted electrons.

\section{INTRODUCTION}

The test facility ELISE (Extraction from a Large Ion Source Experiment) represents an important step in the European R\&D roadmap towards the neutral beam heating systems at the international fusion experiment ITER $^{1,2}$. ELISE provides early experience of the performance and operation of large RF-driven negative hydrogen ion sources with plasma illumination of a source area of $1 \times 0.9 \mathrm{~m}^{2}$ and an extraction area of $0.1 \mathrm{~m}^{2}$ using 640 apertures, i.e. the source area of a half-size ITER source. Consequently, the test facility is aimed to demonstrate the large-scale extraction and acceleration of negative hydrogen and deuterium ions with currents of half the value required for the ITER beam line. The accelerated current to be achieved is $23 \mathrm{~A} \mathrm{H}^{-}$and $20 \mathrm{~A} \mathrm{D}^{-}$stable for up to one hour. Beside the requirement on the extracted current densities of $329 \mathrm{~A} / \mathrm{m}^{2} \mathrm{H}^{-}$and $286 \mathrm{~A} / \mathrm{m}^{2} \mathrm{D}^{-}$, the ratio of co-extracted electrons to ions must be kept below one $^{2}$. In particular the latter requirement is quite demanding as has been shown on the ITER RF prototype sources (source area of $1 / 8$ of the ITER source) ${ }^{3,4}$. Another quite demanding requirement concerns the beam uniformity of these large beams: deviations of $10 \%$ at maximum can be tolerated ${ }^{2}$. As part of the European R\&D roadmap the Neutral Beam Test Facility (NBTF) is currently under construction in Padua, hosting a test facility for the full-size ITER source and the prototype of the Heating Neutral Beam (HNB) for ITER ${ }^{5}$.

The source has to operate at a pressure of $0.3 \mathrm{~Pa}$ or below ${ }^{2}$. In order to generate sufficient negative ions and to reduce the amount of co-extracted electrons, the production of negative ions is based on the surface

\footnotetext{
${ }^{\text {a) }}$ Invited paper published as part of the Proceedings of the 16th International Conference on Ion Source, New York City, NY, August, 2015.

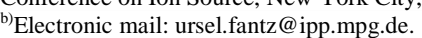

conversion process instead of utilizing the volume mechanisms ${ }^{6}$. For this purpose caesium is evaporated into the source such that the first grid of the extraction system is covered by a caesium layer resulting in a surface with low work function (typically the one of bulk caesium). Here, mainly the impinging hydrogen atoms are converted into negative ions whereas the positive ions are mandatory to compensate the negative ion charge in the boundary layer and to minimize the depth of an arising potential well ${ }^{7}$. Caesium evaporation imposes the challenge to achieve temporally stable and uniform caesium layers over the large grid size in order to meet the ITER requirements in terms of stable and uniform current densities ${ }^{8}$.

The paper reports on the latest ELISE achievements using caesium with focus on extension of the pulse length in hydrogen and in deuterium.

\section{THE ELISE TEST FACILITY}

The ion source and the extraction system have been designed following as close as possible the ITER design. However, some modifications have been carried out to allow better diagnostic access and to improve considerably the experimental flexibility $9,10,11$. After three years of construction and assembly ELISE has been commissioned in Nov. 2012 with first plasma pulses in Feb. 2013.

Figure 1 shows the ion source with a view on the four RF cylindrical drivers in which the plasma is generated via inductive coupling. Each of the two $1 \mathrm{MHz}$ RF generators with a RF power of maximum $180 \mathrm{~kW}$ supplies two horizontal drivers connected in series. The plasma expands from the drivers into the source chamber and illuminates the grid system (Figure 1). For evaporation of caesium two dispenser ovens capable to achieve evaporation rates in the order of $10 \mathrm{mg} / \mathrm{h}$ are mounted in side ports in the expansion chamber. 

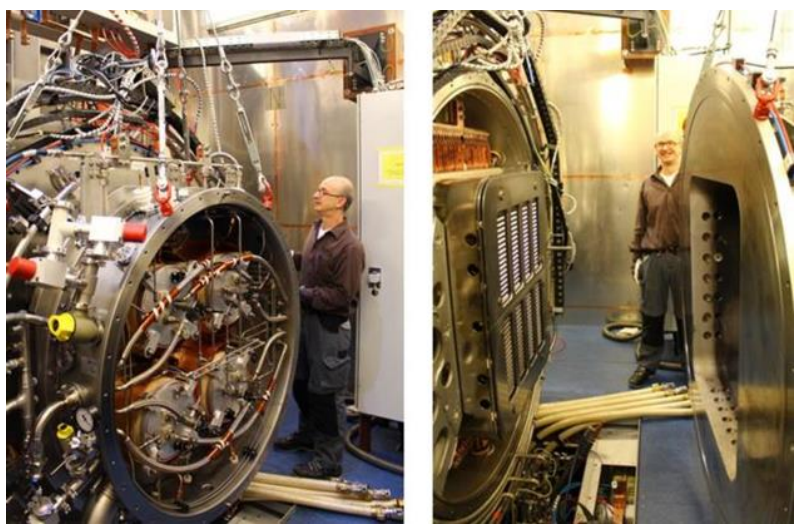

FIG. 1. (Color online). The RF driven ion source at the ELISE test facility. Left. View onto the RF drivers with dismantled vacuum dome. Right. Source opening with view onto the grid system and into the source chamber.

According to the tandem concept of an ion source ${ }^{6}$, a horizontal magnetic filter field is used (i) to cool the electrons down from $\sim 10 \mathrm{eV}$ (driver) to $\sim 1 \mathrm{eV}$ (close to the extraction system) and thus minimize $\mathrm{H}^{-}$destruction by collisions, (ii) to reduce the amount of co-extracted electrons, and (iii) to enhance the extraction probability for surface-produced negative ions ${ }^{8}$. In contrast to the smaller prototype source which allows for using permanent magnets, the field is created by a current up to $5.3 \mathrm{kA}$ flowing vertically through the plasma grid $^{12}$. In the present configuration, with the return conductors located between the drivers, the conversion factor between PG current and field strength at the center of the plasma grid is $1 \mathrm{mT} / 1.06 \mathrm{kA}$.

The extraction system is designed for acceleration of negative hydrogen ions up to $60 \mathrm{kV}$ with an extraction voltage of $10 \mathrm{kV}$. Plasma operation up to one hour is feasible, but extraction is only possible in a pulsed mode (10 s every $\sim 150 \mathrm{~s}$ ) due to the limits of the available HV system. The grid system consists of three grids: the plasma grid (PG) which separates the plasma from the beam region, the extraction grid (EG), where the co-extracted electrons are filtered out of the beam by embedded magnets, and the grounded grid (GG). Each grid has 640 extraction apertures grouped in $5 \times 16$ apertures. The grids consist of two segments in vertical arrangement, i.e. top and bottom segment. The EG segments are insulated against each other so that the current flowing from each segment can be measured individually. In order to suppress the amount of co-extracted electrons, the plasma grid is positively biased with respect to the source.

ELISE is equipped with source diagnostics, mainly optical emission spectroscopy and pin probes using ports close to the extraction system ${ }^{13}$. A set of beam diagnostic tools provides information about the large ion beam: a tungsten wire calorimeter is used for beam monitoring during operation, whereas quantitative parameters such as divergence and uniformity are obtained from beam emission spectroscopy using 20 lines-of-sight and a dedicated diagnostic calorimeter ${ }^{14}$.

\section{RESULTS}

\section{A. Achievements in short pulses}

The very first results obtained from the caesiated source are summarized by Franzen ${ }^{15}$. Operation was carried out in hydrogen and deuterium at low RF power $(20 \mathrm{~kW} /$ driver) to gain experience with source operation and to keep the amount of co-extracted electrons low. The maximum tolerable heat load of the co-extracted electrons on one segment of the EG was set to $75 \mathrm{~kW}$ although the system is designed to handle $200 \mathrm{~kW}$. The plasma pulse length was $20 \mathrm{~s}$ with one extraction phase (beam blip) of $10 \mathrm{~s}$, i.e. the typical short-pulse operation mode. First attempts have been made to enhance the total pulse length, i.e. having several beam blips with about $150 \mathrm{~s}$ pause in between.

The ITER-relevant results by increasing the RF power to a maximum of $55 \mathrm{~kW} /$ driver in the short-pulse mode and a filling pressure of $0.3 \mathrm{~Pa}$ are shown in Figure 2. For higher power levels results from the prototype source at the test facility BATMAN (6 s plasma with $4 \mathrm{~s}$ beam) are included $^{3}$. All data are taken at extraction voltages between $8.5 \mathrm{kV}$ and $9.5 \mathrm{kV}$. The ITER requirements of minimum
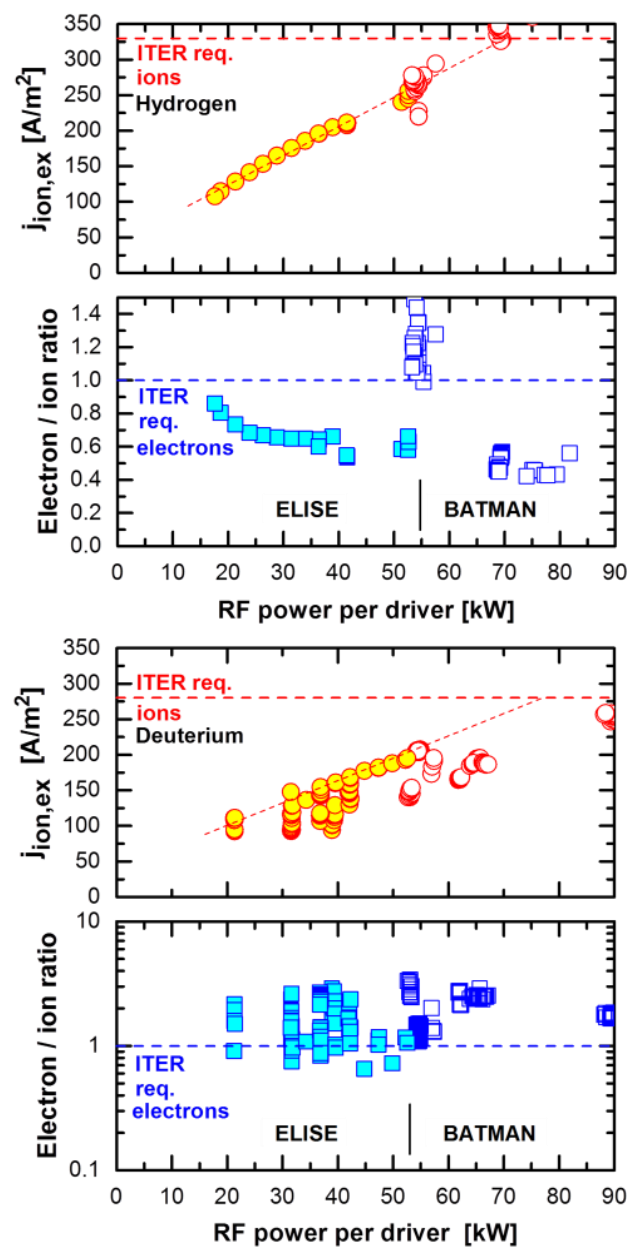

FIG. 2. (Color online). Performance plots for hydrogen and deuterium operation. The open symbols refer to achievements at the test facility BATMAN equipped with a single driver (prototype source). 
extracted current density and maximum tolerable electronto-ion ratio are indicated as well. The results for the extracted current density as a function of the RF power per driver (four drivers at ELISE, one driver at BATMAN) allow for a linear extrapolation to the ITER requirements. For both hydrogen and deuterium, a required RF power of about $80 \mathrm{~kW}$ per driver can be expected for the large source which is less than the power being installed for the ITER source (100 kW/driver).

In hydrogen, operation with a low co-extracted electron current is no issue, neither on BATMAN nor on ELISE, whereas for deuterium the dynamics of the electron-to-ion ratio is much more pronounced and ratios below one are difficult to achieve. For both hydrogen and deuterium, less filter field as expected is needed: only $2.2 \mathrm{mT}$ and $3.3 \mathrm{mT}$ are used at ELISE respectively, whereas the peak value at BATMAN is $7 \mathrm{mT}$. In addition to the different 3-dim topology of the filter field also the integral value along the axial direction from the PG to the driver exit is much lower: about $0.4 \mathrm{mTm}$ (hydrogen) and $0.6 \mathrm{mTm}$ (deuterium) in ELISE compared to $1-1.5 \mathrm{mTm}$ in BATMAN. Increasing the filter field in ELISE results in a simultaneous reduction of the extracted negative ion current density; the same trend is observed when increasing the bias current to reduce the co-extracted electrons. As already known from the prototype source, these trends depend strongly on the caesium conditioning, for which not only the evaporation rate is a free parameter but also the temperatures of the source chamber and the plasma grid. At the present stage of experiments, the latter parameters have been fixed to $40^{\circ} \mathrm{C}$ and $125^{\circ} \mathrm{C}$ respectively. Dedicated temperature variations within 30$60^{\circ} \mathrm{C}$ for the walls and up to $200{ }^{\circ} \mathrm{C}$ for the grid are scheduled in order to optimize the caesium redistribution in the large source.

\section{B. Long pulse operation}

Extension of the pulse length is often limited by the amount of the co-extracted electrons which strongly depends on the caesium dynamics, i.e. the evaporation, distribution and deterioration of the caesium by the background gas in the vacuum phase as well as on the redistribution and cleaning effect of the caesium layers during plasma phases ${ }^{4,8,15}$. As only pulsed extraction is available in time intervals of about $150 \mathrm{~s}$, the pulse lengths were extended first to accommodate two beam blips with the goal to achieve stable pulses before proceeding to pulses with three beam blips. It must be pointed out, that the ions are by far more stable than the electrons. The latter ones reveal a strong increase during $\mathrm{cw}$ extraction as seen at the prototype source of the MANITU test facility ${ }^{3,4}$.

Figure 3 shows results for hydrogen pulses with three beam blips at $0.35 \mathrm{~Pa}$ with electron-to-ion ratios well below one. As expected, the extracted current density of negative ions increases with increased power. A decrease of less than $5 \%$ in the ion current density is observed from the first to the third beam blip, being slightly more pronounced at the higher power level. The electrons,

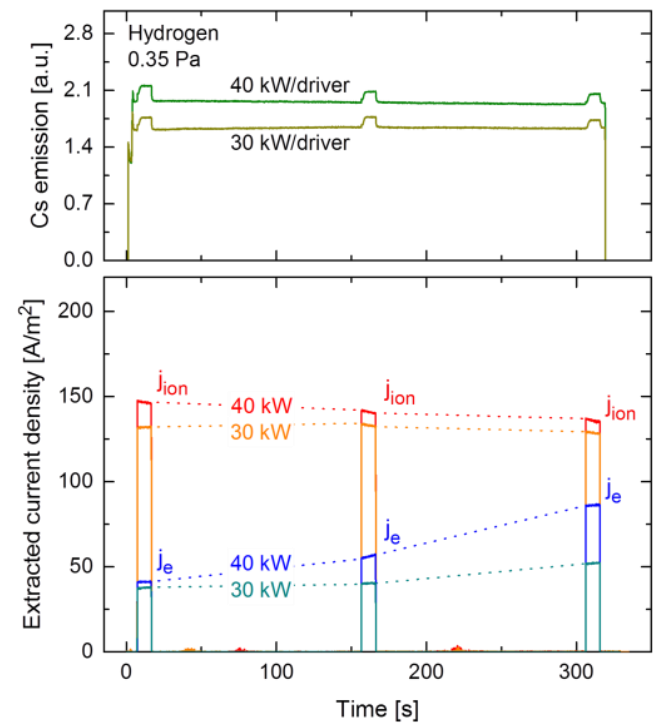

FIG. 3. (Color online). Hydrogen pulses with three beam blips with an extraction voltage of $5 \mathrm{kV}$ for two RF powers.

however, shows a strong dependence on the RF power; the increase with time is much more pronounced at higher power level. Interestingly, the currents obtained in the individual beam blips follow clearly a trend line as indicated by the dotted curve. The time trace of the caesium signal $(852 \mathrm{~nm}$ line of neutral caesium observed via a line-of-sight parallel to the PG at $2 \mathrm{~cm}$ distance using photodiode with interference filter) reveals a pronounced increase during extraction. The increase is attributed to back-streaming positive ions from the accelerator, which hit the back plate of the source and consequently sputter caesium from this surface. At $30 \mathrm{~kW} /$ driver the caesium signal is stable during plasma-only time (from beam blip to beam blip), whereas a slight decrease is obtained for the higher power level.

The best $400 \mathrm{~s}$ pulse at a power level of $45 \mathrm{~kW} /$ driver

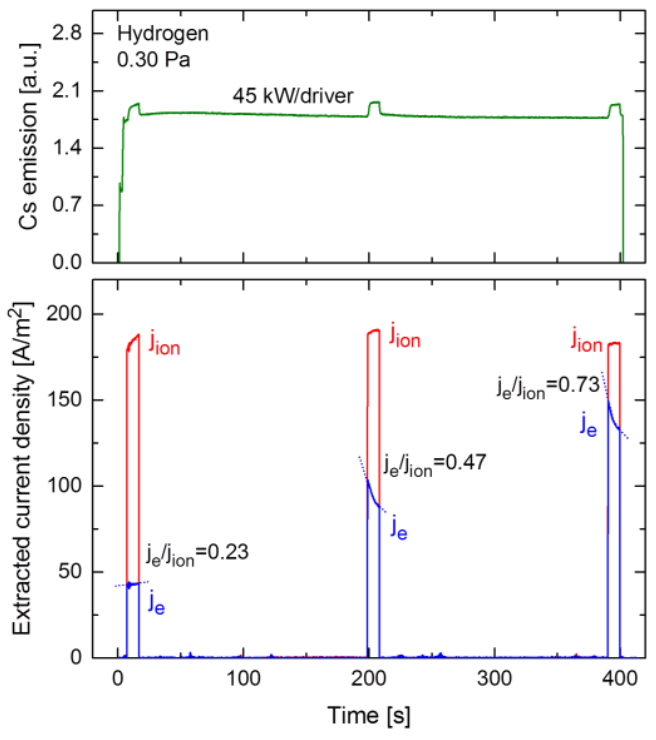

FIG. 4. (Color online). Hydrogen pulse with three beam blips at an $\mathrm{RF}$ power of $45 \mathrm{~kW} /$ driver $(180 \mathrm{~kW}$ in total). 
with an extraction voltage of $7 \mathrm{kV}$ is shown in Figure 4. The extracted current density is above $183 \mathrm{~A} / \mathrm{m}^{2}$ corresponding to a current of 18.3 A with a variation of $5 \%$. The electron-to-ion ratio is well below one, however the electron current reveals a strong temporal dynamic not only from beam blip to beam blip (indicated by dotted lines) but also during the beam blip. Obviously, a deconditioning of the caesium takes place between the pulses, whereas the caesium released by the back streaming ions improves the source performance. Attempts to increase the caesium evaporation rate and thus supply more caesium to the source had the disadvantage to cause either breakdowns between the grids or to dilute the driver plasma such that RF matching becomes a problem.

The high and unpredictable dynamics of the coextracted electron currents in long pulses prevents an increase of the RF power as this is unavoidably correlated with an increase of the amount of co-extracted electrons.

Compared to hydrogen, in deuterium the control of coextracted electrons is more difficult and limits the parameter range for increasing RF power and pulse lengths. Figure 5 shows one of the best deuterium pulses at $0.3 \mathrm{~Pa}$ using an extraction voltage of $5 \mathrm{kV}$. The coextracted electrons are well below the ions at the first beam blip, but increase by a factor of two at the next beam blip such that the ratio is above one. As for hydrogen (Figure 4), the trend during the extraction reverses as caesium is released from the back plate. The caesium signal shows a pronounced decrease over the length of the plasma pulse. It should be noted, that a different line-ofsight has been used to detect caesium such that the values in deuterium cannot be compared with the ones for hydrogen. In general, deuterium operation requires more caesium, i.e. a higher evaporation rate; typically a factor of two higher neutral caesium density is measured in deuterium than in hydrogen ${ }^{8,15}$.

In contrast to hydrogen campaigns, for which usually the caesium conditioning starts with a cleaned (almost
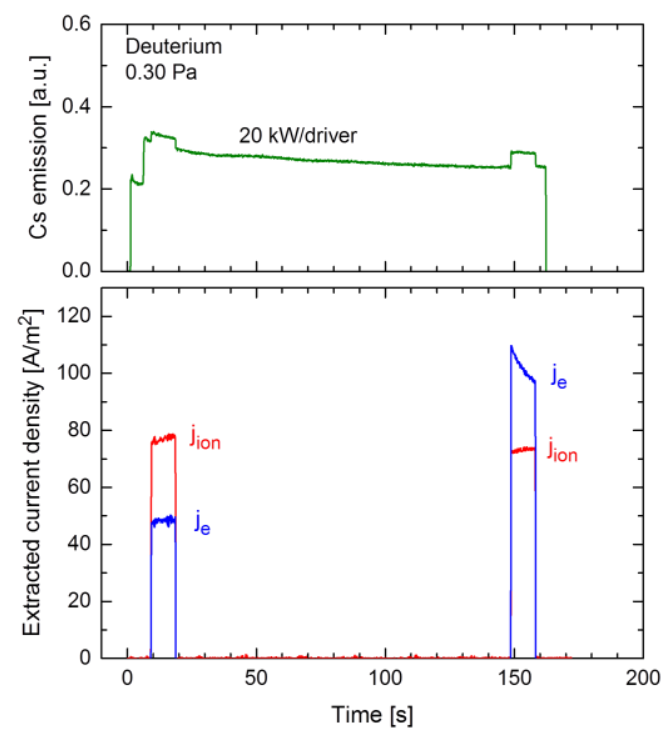

FIG. 5. (Color online). Deuterium pulse with two beam blips at low RF power (20 kW/driver, i.e. $80 \mathrm{~kW}$ in total). caesium-free) source, deuterium operation usually follows the hydrogen campaign. This means the source is well caesium conditioned for hydrogen before caesium conditioning in deuterium starts. For deuterium, however, more caesium is needed and the filter field has to be increased to suppress the co-extracted electrons. This changes the redistribution and consequently the caesium dynamics in the ion source. Several caesium conditioning scenarios have been tested in deuterium. The same procedure as in hydrogen failed: conditioning at higher pressure and short pulses and then successively reducing the pressure and increasing the pulse length. Therefore, long plasma phases without extraction have been tested to support the caesium redistribution. Plasma pulses have been performed with and without bias, with the purpose to support the flux of singly charged positive caesium ions towards the grid in the latter case (without bias). The plasma grid temperature has been decreased to $80^{\circ} \mathrm{C}$ to reduce the thermal desorption of caesium from the grid. Any measure to increase the caesium flux towards the grid in the vacuum phase (higher temperatures, higher evaporation rates) results in a loss of the high voltage holding between the grids. Up to now, caesium conditioning at low pressure, i.e. directly at $0.3 \mathrm{~Pa}$, with longer vacuum phases (up to one hour; standard time is about 4 minutes) between the pulses and a slightly higher evaporation rate has been the most successful scenario. This is attributed to the built up of caesium reservoirs in the plasma chamber which are then redistributed by the plasma; an effect already observed in the smaller prototype sources ${ }^{4,8}$. Although an optimized recipe has not been identified yet, there are parameters which can be tested and optimized, such as the combination of bias and strength of the filter field, the combination of source and grid temperature, the positioning of the caesium oven, the direction of the nozzle of the caesium oven, different magnetic filter field configurations, etc..

Nevertheless, for the next experimental campaign, which starts after a maintenance phase and cleaning of caesium from the source chamber, it is planned to start with the caesium conditioning in deuterium. This might give the optimum caesium distribution in the source right from the beginning and might simplify the identification of reliable caesium conditioning.

\section{One hour pulse in hydrogen}

In hydrogen effort has been taken to extend the pulse length towards one hour, i.e. the pulse length envisaged for ITER NBI. Limited by the amount of co-extracted electrons, a low RF power has been chosen $(20 \mathrm{~kW} /$ driver $)$ and the extraction voltage was set to $6 \mathrm{kV}$ only. The PG current for the magnetic filter field has been slightly increased to $2.8 \mathrm{kA}$ and the bias current was set to $20 \mathrm{~A}$, resulting in a bias potential of about $22 \mathrm{~V}$. At these parameters very stable $400 \mathrm{~s}$ pulses (in terms of electron currents) have been achieved easily and reproducibly.

The extracted current densities of the ions and the electrons as well as the electron-to-ion ratio of the record- 

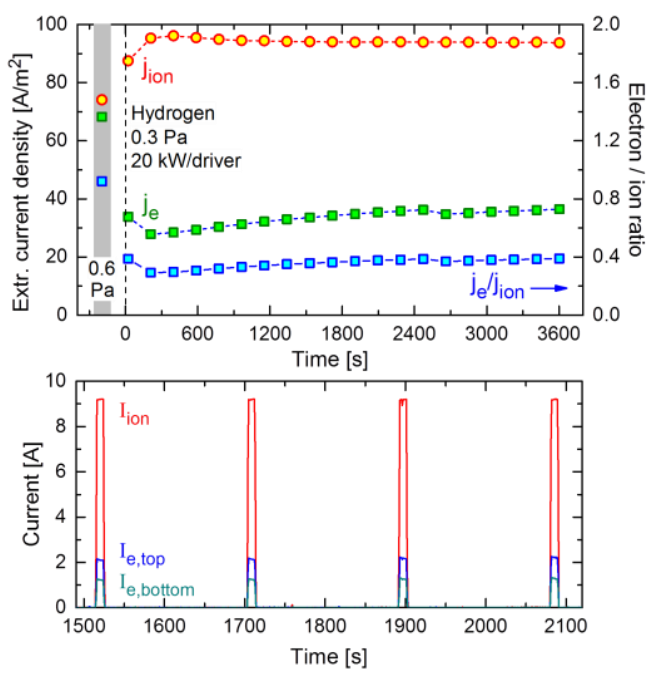

FIG. 6. (Color online). One hour hydrogen pulse at $0.3 \mathrm{~Pa}$ with $20 \mathrm{~kW} /$ driver after a $20 \mathrm{~s}$ pulse at $0.6 \mathrm{~Pa}$ (the first pulse of the day).

setting one hour pulse are shown in Figure 6. The data of the preceding short pulse at $0.6 \mathrm{~Pa}$ is plotted as well. It was the first pulse of this operational day in which also caesium evaporation was switched off. Caesium conditioning took place the day before. The stable performance achieved for the one hour pulse means either that the caesium reservoir within the ion source was sufficient and the redistribution very reproducible or that the caesium layer on the plasma grid was very persistent. Nevertheless, it demonstrates that a stable 1 hour pulse is possible without additional caesium evaporation and that such a pulse can follow directly a short pulse (20 s plasma with $10 \mathrm{~s}$ beam). Furthermore it demonstrates that a long re-conditioning time of the source is not mandatory in a well caesiated source.

The average value of the extracted ion current density obtained in the 20 beam blips is $93 \mathrm{~A} / \mathrm{m}^{2}$ with a deviation of $5 \%$ resulting in a total ion current of $9.3 \mathrm{~A}$. The electron-to-ion ratio is below 0.4 ; the co-extracted electron current increases slightly from one beam blip to the other except for the first two beam blips. The time traces of the currents in the beam blips no. 9 to 12 (Figure 6) show the stability of the ion current and also the stability of the electron currents during the beam blip. As mentioned before, the current onto the upper and lower segment of the EG is measured separately $\left(\mathrm{I}_{\mathrm{e}, \text { top }}\right.$ and $\left.\mathrm{I}_{\mathrm{e}, \mathrm{bottom}}\right)$. Typically the current measured in the upper segment is higher than the one in the lower segment, which is also the case in this pulse.

The beam is dumped on the specially designed diagnostic calorimeter allowing for the determination of the accelerated current density via water calorimetry ${ }^{14,15}$. In this pulse about $82 \%$ of the extracted ion current is measured on the calorimeter. An infrared (IR) camera is used to obtain images of the beam on the diagnostic calorimeter which is blackened by a thin layer of $\mathrm{MoS}_{2}$ for this purpose (emissivity about 83\%). An example of an image taken from the last time frame of the IR camera

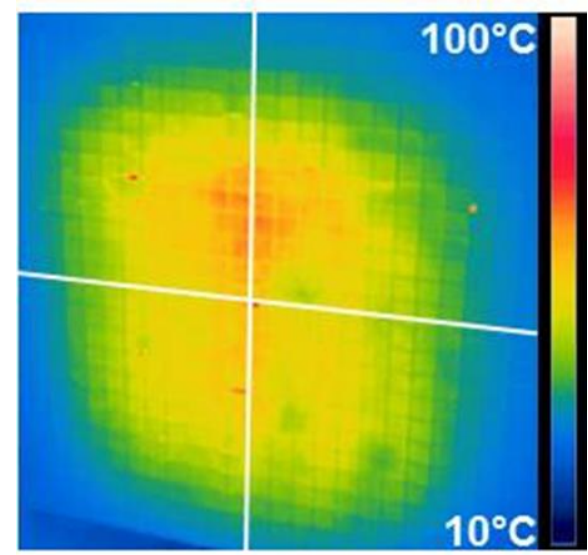

FIG. 7. (Color online). Image of the IR camera with a view onto the diagnostic calorimeter taken in the $10^{\text {th }}$ beam blip (at about $1800 \mathrm{~s}$ ) of the one hour pulse (Figure 6). The lines indicate the position of the beam center at the calorimeter.

within the beam blip at $1700 \mathrm{~s}$ is shown in Figure 7. Due to the viewing geometry the image is slightly distorted; the nominal center lines are indicated in the figure for orientation. Good beam homogeneity is observed with slightly higher intensity in the upper part of the calorimeter than in the lower part. The beam is shifted downwards which is caused by the magnetic filter field in the acceleration system. The calorimeter is in $3.5 \mathrm{~m}$ distance from the last grid, the GG, resulting in a beam shift of several centimeters, mostly depending on the strength of the filter field.

The analysis of the Doppler-shifted $\mathrm{H}_{\alpha}$ peaks obtained by the 20 lines-of-sight of the beam emission spectroscopy reveals an inhomogeneity of about $3 \%$ in horizontal direction and about $7 \%$ in vertical direction. The beam divergence is determined to be $3.3^{\circ}$. It should be noted that beam divergences between $1^{\circ}$ and $2^{\circ}$ are measured in good perveance conditions ${ }^{15}$. For such cases the individual beamlet groups are resolved in the IR image.

Although the results are obtained at quite low RF power, they are very promising for fulfilling the ITER requirements. At the chosen $20 \mathrm{~kW} /$ driver a current of 9.3 A was obtained which means by extrapolating linearly towards the $80 \mathrm{~kW} /$ driver derived from the short pulse operation (Figure 2), the extracted current would be $37 \mathrm{~A}$ and thus about $12 \%$ higher than the target value. Concurrently, the amount of co-extracted electrons must be well controlled, as the co-extracted electron current might increase more than linearly with power.

The next experimental campaigns are aimed to demonstrate the ITER requirements with focus on long pulse operation at higher RF power (up to $80 \mathrm{~kW} /$ driver) and extraction voltages of $10 \mathrm{kV}$. This is in particular a challenge for deuterium which reveals a much stronger dynamics of the co-extracted electrons than hydrogen.

\section{CONCLUSIONS}

Since the first plasma pulses in Feb. 2013 steady progress is obtained on the test facility ELISE which is 
aimed to meet the requirements of the ion source for the neutral beams on ITER but at half the source area as for ITER.

The results in short-pulse operation (20 s plasma, $10 \mathrm{~s}$ beam) obtained in a RF power range of $20-55 \mathrm{~kW} /$ driver, i.e. $80-220 \mathrm{~kW}$ in total, allow to extrapolate to $80 \mathrm{~kW} /$ driver needed to obtain the required ion current densities in hydrogen and in deuterium. This is quite encouraging as it is below the installed $100 \mathrm{~kW} /$ driver at the ITER source. In hydrogen, the amount of co-extracted electrons is often well below one but is of major concern in deuterium. This reveals the same behavior as known from the smaller prototype sources. Nevertheless, much lower magnetic filter field strengths are needed in ELISE than in the prototype sources. This is attributed to the different 3dim topology of the magnetic filter field. Similarly to the prototype source, higher field strengths are needed in deuterium to suppress the electrons.

The extension of the pulse length towards one hour required a careful caesium conditioning. To keep the coextracted electrons at the tolerable level, the RF power and the extraction voltage have been kept at a low level. Focus is laid on achieving stable pulses at $0.3 \mathrm{~Pa}$. For hydrogen a stable 1 hour plasma discharge with repetitive $10 \mathrm{~s}$ beam blips was demonstrated with 9.3 A extracted ion current and an electron-to-ion ratio of 0.4 using a $20 \mathrm{~kW} /$ driver, which is less than a quarter of the available RF power. At $45 \mathrm{~kW}$ RF power per driver and thus half of the available RF power, a stable $400 \mathrm{~s}$ plasma discharge with extracted currents in the beam blips of $18.3 \mathrm{~A}$ at an electron-to-ion ratio of 0.7 could be achieved. Challenges in the long pulse operation are the caesium dynamic in the source and the stability of the co-extracted electron current, the latter being the limiting parameter for the power load on the extraction grids and thus for the source performance, in particular in deuterium.

In the scheduled maintenance phase, which followed these experimental campaigns, no major issues have been identified at the individual components. For the next experimental campaign the source chamber has been cleaned from caesium. It is planned to start with the caesium conditioning in deuterium. Focus will be laid on long pulse operation at higher RF power (up to $80 \mathrm{~kW} /$ driver) and operation at extraction voltage of up to $10 \mathrm{kV}$.

\section{ACKNOWLEDGMENTS}

The work was supported by a contract from Fusion for Energy (F4E-2009-0PE-32-01), represented by Antonio Masiello, with an amount of $4 \mathrm{M} €$. The opinions expressed herein are those of the authors only and do not represent the Fusion for Energy's official position.

This work has been carried out within the framework of the EUROfusion Consortium and has received funding from the Euratom research and training programme 20142018 under grant agreement No 633053. The views and opinions expressed herein do not necessarily reflect those of the European Commission.
${ }^{1}$ A. Masiello, G. Agarici, T. Bonicelli, M. Simon, V. Antoni, H. De Esch, A. De Lorenzi, M. Dremel, P. Franzen, R. Hemsworth, M. Liniers, D. Marcuzzi, D. Martin, R. Piovan, A. Simonin, P. Sonato, E. Surrey, L. Svensson, A. Tanga, V. Toigo, C. Waldon, and P. Zaccaria, Fusion Eng. Des. 86, 860 (2011).

${ }^{2}$ R. Hemsworth, H. Decamps, J. Graceffa, B. Schunke, M. Tanaka, M. Dremel, A. Tanga, H.P. De Esch, F. Geli, J. Milnes, T. Inoue, D. Marcuzzi, P. Sonato, and P. Zaccaria, Nucl. Fusion 49, 045006 (2009).

${ }^{3}$ E. Speth, H.D. Falter, P. Franzen, U. Fantz, M. Bandyopadhyay, S. Christ, A. Encheva, M. Fröschle, D. Holtum, B. Heinemann, W. Kraus, A. Lorenz, Ch. Martens, P. McNeely, S. Obermayer, R. Riedl, R. Süss, A. Tanga, R. Wilhelm and D. Wünderlich, Nucl. Fusion 46 S220-S238 (2006).

${ }^{4}$ W. Kraus, U. Fantz, P. Franzen, M. Fröschle, B. Heinemann, R. Riedl, and D. Wünderlich, Rev. Sci. Instrum. 83, 02B104 (2012).

${ }^{5}$ V. Toigo et al., Nucl. Fusion 55, 083025 (2015).

${ }^{6}$ M. Bacal and M. Wada, Applied Physics Reviews 2, 021305 (2015).

${ }^{7}$ D. Wünderlich, L. Schiesko, P. McNeely, U. Fantz, P. Franzen and the NNBI-Team, Plasma Phys. Control. Fusion 54, 125002 (2012).

${ }^{8}$ U. Fantz, P. Franzen, D. Wünderlich, Chemical Physics 398, 7 (2012).

${ }^{9}$ B. Heinemann, H. Falter, U. Fantz, P. Franzen, M. Fröschle, R. Gutser, W. Kraus, R. Nocentini, R. Riedl, E. Speth, A. Stäbler, D. Wünderlich, P. Agostinetti, and T. Jiang, Fusion Eng. Des. 84, 915 (2009).

${ }^{10}$ B. Heinemann, H.-D. Falter, U. Fantz, P. Franzen, M. Froeschle, W. Kraus, C. Martens, R. Nocentini, R. Riedl, E. Speth, and A. Staebler, Fusion Eng. Des. 86, 768 (2011).

${ }^{11}$ R. Nocentini, U. Fantz, P. Franzen, M. Fröschle, B. Heinemann, R. Riedl and the NNBI Team, IEEE Trans. Plasma Sci. 42, 616 (2014).

${ }^{12}$ U. Fantz, P. Franzen, W. Kraus, L. Schiesko, C. Wimmer, and D. Wünderlich, AIP Conference Proceedings 1655, 040001 (2015).

Sci. Instrum. 84, 093102 (2013).

${ }^{13}$ D. Wünderlich, U. Fantz, P. Franzen, R. Riedl, and F. Bonomo, Rev. Sci. Instrum. 84, 093102 (2013).

${ }^{14}$ R. Nocentini, U. Fantz, P. Franzen, M. Froeschle, B. Heinemann, R. Riedl, B. Ruf, D. Wuenderlich, the NNBI Team, Fusion Eng. Des. 88, 913 (2013).

${ }^{15}$ P. Franzen, U. Fantz, D. Wünderlich, B. Heinemann, R. Riedl, W. Kraus, M. Fröschle, B. Ruf, R. Nocentini and the NNBI Team, Nucl. Fusion 55053005 (2015). 